\title{
Rearing Effects on Cerebrospinal Fluid Oxytocin Concentration and Social Buffering in Rhesus Monkeys
}

\author{
James T Winslow*, 1,2,3, Pamela L Noble ${ }^{2}$, Casie K Lyons ${ }^{2}$, Sheila M Sterk ${ }^{2}$ and Thomas R Insel Th $^{2,3}$ \\ 'Yerkes National Primate Research Center, Atlanta, GA, USA; ${ }^{2}$ Center for Behavioral Neuroscience, Atlanta, GA, USA; ${ }^{3}$ Psychiatry and Behavioral \\ Biology, Emory University Medical School, Atlanta, GA, USA
}

\begin{abstract}
Mother-reared (MR) and nursery-reared (NR) male rhesus monkeys exhibit profound and persistent differences in social and emotional behavior. Compared to MR animals, NR monkeys show reduced reciprocal social behaviors and increased agonistic behavior and high levels of stereotypy. Cerebrospinal fluid oxytocin (CSF OT) in NR monkeys was significantly reduced compared to MR monkeys measured at 18, 24, and 36 months of age. Correlations between OT and individual social behavioral profiles measured across rearing conditions also revealed a significant association between OT and the expression of affiliative social behaviors including allogrooming and reciprocal intermale mounting at each age examined. In contrast, CSF vasopressin levels did not differ according to rearing history, but did correlate with fearful behaviors independent of rearing history. Differential rearing was not associated with differences in basal or stressrelated plasma cortisol, although these levels did progressively decline as monkeys matured. MR but not NR monkeys were able to use a social companion to buffer their response to a stressor, but OT levels did not appear to be sensitive to the presence of a social companion in either group. These results are consistent with earlier reports from studies of rodents suggesting an important role for central OT pathways in the development of social affiliation.

Neuropsychopharmacology (2003) 28, 9 I0-918, advance online publication, 26 March 2003; doi: I 0. I038/sj.npp. I 300 I 28
\end{abstract}

Keywords: cerebrospinal fluid; oxytocin; vasopressin; cortisol; adverse rearing; macaca mulatta

\section{INTRODUCTION}

While recent studies have reported genes for social behavior (reviewed in Insel and Young, 2000), nearly 50 years of research has documented the powerful effects of rearing environment on adult social interaction. Birds reared by parents of another species fail to develop species-typical calls (Immelmann, 1969). In rats, variations in rearing environment ranging from normative differences in maternal licking and grooming to more extreme interventions such as social isolation are associated with altered stress responsiveness and changes in social behavior in adulthood (Hall et al, 1998; Liu et al, 2000). Human infants raised in socially deprived environments show decreased social interaction as children, exhibiting some of the features of autism (Rutter et al, 1999). As a particularly close model of this human syndrome, rhesus monkeys reared in a nursery away from the mother manifest deficits in social interaction

*Correspondence: Dr JT Winslow, Yerkes National Primate Research Center, Emory University, 954 Gatewood Road NE, Atlanta, GA 30322, USA, Tel: + I 404727 7728, Fax: + I 404727 7845,

E-mail: jwinslow@rmy.emory.edu

Received 20 September 2002; revised 18 November 2002; accepted 17 December 2002

Online publication: 19 December 2002 at http://www.acnp.org/ citations/Npp 121902445 and increased self-directed behaviors. First described by Harlow (Harlow et al, 1955; Harlow and Harlow, 1962; Harlow and Suomi, 1974), many laboratories have replicated the social behavior deficits of rhesus monkeys raised in a nursery (Champoux et al, 1991; Sackett, 1984; Suomi et al, 1971).

Although we have extensive research documenting the behavioral deficits of nursery-reared monkeys, we know relatively little about associated neurobiological and neuroendocrine correlates (Ginsberg et al, 1993a, b; Sanchez et al, 1998; Siegel et al, 1993) These neural changes are of interest not only because of their potential relevance to autism but because they may indicate how normal maternal-infant interactions support neural development. The current study was undertaken as a longitudinal comparison of monkeys in two rearing conditions: nursery-rearing (NR) $v s$ seminaturalistic mother-rearing (MR). Specifically we planned to test the following three hypotheses:

1. Based on recent studies of the role of neurohypophyseal peptides in social affiliation of rodents (Insel, 1992), these two groups of monkeys will differ in central concentrations of oxytocin (OT) and vasopressin (AVP).

2. Based on earlier data of rearing effects on stress responsiveness, these two groups of monkeys will differ 
in their plasma concentrations of cortisol either at baseline or following a stressor.

3. Based on the evidence for deficits in social interaction, these two groups of monkeys will differ in their ability to use a social companion to buffer the effects of a stressful environment.

By studying these monkeys at 18, 24, and 36 months, we tested each of these hypotheses at critical times of development between the juvenile state (18 months) and reproductive maturity ( 36 months). Our results demonstrate that NR have reduced concentrations of central OT and that this reduction is correlated with deficits in social behavior.

\section{METHODS}

\section{Subjects}

A total of 12 male rhesus monkeys (Macaca mulatta, Indian origin) born between April and May 1999 in six large breeding groups maintained at the Yerkes National Primate Research Center Field Station. Infants born to these monkeys were assigned to two different rearing conditions: $\mathrm{NR}$ and MR. All infants were derived from mothers of midlevel social status.

\section{Rearing Conditions}

NR monkeys were removed from their mothers within $48 \mathrm{~h}$ of birth, and raised according to standard nursery protocols at the Yerkes Main Station facility. Accordingly, infants were individually housed for the first 45-60 days and bottle fed by nursery care staff. Each infant was then pairhoused with another of the NR subjects, forming three social pairs. These pairs were continuously housed together during the day except for 4- to 6-h intervals each day (between 10:00 am and 4:00 pm) when they were separated for individualized feeding and bottle training. At approximately 3 months of age individualized feeding was discontinued and the animals remained pair housed in the nursery facilities without interruption throughout the remainder of the first year.

MR monkeys were recruited from the same birth cohort as NR monkeys. The criterion for the selection of these infants included age matching for NR monkeys, midlevel social status, and no prior history of veterinary intervention requiring maternal separation during the first year of life. At approximately 1 year of age they were removed from their natal groups, transported to the Yerkes Main Station, and housed with a familiar companion. Following a 4-week quarantine, these social pairs along with the cohort of NR monkeys were transferred to a new housing room located at the Yerkes Main Station.

Each animal was housed in a $1.3 \mathrm{~m}^{3}$ cage equipped with a removable, porous (9-5 $\mathrm{cm}$ diam holes) partition to control social contact with the adjacent cage. Members of each pair of monkeys were routinely separated by the partition at approximately 5:00 pm each day. The partition was removed each morning at approximately 9:00 am to permit unrestricted social contact through the day. This process facilitated a predictable pattern of vigorous social interaction each morning.

\section{Assessment of Social Behavior}

Videocage. A custom-built videocage $(1.5 \mathrm{~m}$ high $\times 1.5 \mathrm{~m}$ wide $\times 0.75 \mathrm{~m}$ deep) was constructed to permit videotaping of social pairings. This cage was equipped with casters to permit mobility and two $20^{\prime \prime}$ shelves, one high, the other low. Panels could be inserted at the end of each shelf to separate the animals if necessary. A guillotine door was located on each end of the cage to permit access directly from the home cages. The front of the cage was covered with clear Plexiglas panels and the remainder of the cage was covered in wire mesh.

Round-robin pairings of differentially reared monkeys. At 18,24 , and 36 months of age, every monkey was recruited for a 3- to 4-week study of comparative social interactions with an unfamiliar NR (uNR), unfamiliar MR (uMR), or home-cage (HC) partner in the videocage. Each 1-h observation of pair interactions was separated by 1 week and the order of pairing with different partner types (uNR, uMR, HC) was systematically varied. Each subject consequently was videotaped for a total of $3 \mathrm{~h}$. All videotaped sessions were conducted in the colony housing room.

At 1 week after completion of each round-robin study, samples of blood and cerebrospinal fluid (CSF) were collected from each subject to assess basal measures of cortisol and CSF neuropeptides.

\section{Novel Cage and Social Buffering Test}

At approximately 37 months of age, beginning 1-2 weeks after basal plasma and CSF samples were collected following round-robin pairings, novel-cage stress challenges were conducted to determine if there was a differential response between the two rearing groups. Stress-related blood and CSF samples were collected between 9:30 and 10:30 am. Each animal was tested once per week in one of the three conditions and the order of testing was systematically varied between animals. For basal levels, each subject was administered anesthetic in his $\mathrm{HC}$ and immediately transferred to an adjacent treatment room for biofluid collection. In the alone condition, each animal was first transferred to a cage located in a novel room for $30 \mathrm{~min}$, and then anesthetized before biofluid collection. In the companion condition, each animal along with his cage mate was first transferred to a cage located in a novel room for $30 \mathrm{~min}$, then both were anesthetized for biofluid collection. Behavior was videorecorded and subsequently coded for analysis.

\section{Ethogram}

A comprehensive ethogram was adapted (Altmann, 1962; Hinde and Spencer-Booth, 1967) to cover a range of social and nonsocial behaviors. Briefly, all bouts of motor and stationary activity, agonistic, and nonagonistic social behaviors and vocalizations were coded for real-time computer-assisted data entry. Definitions were designed to provide a comprehensive and exhaustive description of social and nonsocial behaviors. Motor and social behavior categories were not mutually exclusive. Total frequencies and/or durations for each behavioral element were com- 
puted as appropriate and expressed as the rate or total time per hour observation. Social behaviors were further grouped according to affiliative, aggressive, and defensive behaviors. Affiliative behaviors include measures of allogrooming, genital inspection, sitting in contact, clinging, touching, and intermale mounting. Intermale mounting was included in this category based on the interpretation of Reinhardt et al (1986). Aggressive behaviors included cageshaking, hitting, wrestling, biting attack, and open mouth threats. Elements included in defensive behavior included fear grimace, defensive crouch, escape behavior, and screaming. A category designed to quantify time spent engaged in solitary behaviors included self-grooming, object manipulation, solitary stationary-alert, and solitary walking. Abnormal or stereotyped behaviors included thumb sucking, self-biting, and other idiosyncratic, highly repetitive motor patterns.

Videotapes were scored using focal animal techniques (Altmann, 1974) with commercially available computerassisted data collection technology (Noldus, Observer ${ }^{\mathrm{TM}}$ ).

\section{Observer Training and Reliability}

Multiple observers were trained to intra- and interobserver reliabilities of $\geqslant 80 \%$ for frequency and duration of social and motor behaviors on a predesignated $60 \mathrm{~min}$ training tape. Additional reliability at $>80 \%$ was obtained for $2-$ 30 min segments from different tapes from each observation set selected to represent vigorous social interactions (ie 18, 24,36 months).

\section{CSF and Plasma Samples}

Approximately 1 week following completion of each roundrobin behavioral study, each subject was administered doses of ketamine $(5 \mathrm{mg} / \mathrm{kg})$ and Telazol $(4-5 \mathrm{mg} / \mathrm{kg})$ in his HC and immediately transferred to an adjacent treatment room for blood and CSF fluid collection. Blood was drawn from the femoral vein, and CSF was obtained from the cisterna magna. Baseline blood and CSF samples were collected between 9:30 and 10:30 am. For all studies, blood was transferred to EDTA-coated tubes and stored on ice, centrifuged at $4^{\circ} \mathrm{C}$, and plasma aliquots stored at $-80^{\circ} \mathrm{C}$. Cisternal CSF samples were immediately frozen on crushed dry ice and subsequently stored at $-80^{\circ} \mathrm{C}$. Unless otherwise indicated, the interval from initial contact to initial blood collection was less than $5 \mathrm{~min}$ and for CSF less than $10 \mathrm{~min}$.

\section{Hormone Determinations}

Unextracted CSF samples $(20 \mu \mathrm{l})$ were assayed in duplicates for OT and AVP immunoreactivity by RIA. Plasma were diluted in a 5:1 ratio with assay buffer and assayed in duplicate for OT. No further extraction was applied. Determinations were prepared by the Yerkes National Primate Research Center Endocrine Core Laboratory using commercially prepared kits produced by R\&D Systems (Minneapolis, MN; cat. DE-1900 (EIA kit)). The sensitivity of assays was $<5.0 \mathrm{pg} / \mathrm{ml}$ for OT and $<4.0 \mathrm{pg} / \mathrm{ml}$ for AVP. Crossreactivity of the OT assay for AVP was $<0.001$. Crossreactivity of the AVP assay for OT was $<0.001$. The intra- and interassay coefficients of variation at the relevant concentrations in each assay were approximately $10 \%$.

OT levels in CSF were measured at 18,24 , and 36 months of age. AVP levels were only measured at 18 and 24 months of age.

Plasma cortisol determinations were prepared using a commercially prepared kit (Diagnostic Systems Laboratories, Webster, TX; cat. DSL-2000). The range for this assay at $10 \mu \mathrm{l}$ was $1.25-150 \mu \mathrm{g} / \mathrm{dl}$. The intra- and interassay coefficients of variation at the relevant concentrations in each assay were $<10 \%$.

\section{Statistical Analyses}

To compare behavioral scores during play sessions we used a multifactor ANOVA with repeated measures: a betweensubject factor was used for rearing history (MR $v s \mathrm{NR}$ ) and repeated measures included partner type (HC, uNR, and uMR) and age (18, 24, and 36 months). For CSF neuropeptide levels, two-way ANOVAs were used to assess the rearing history (between-subjects factor) and age (within-subjects factor). Where appropriate, post hoc Newman-Keuls Test comparisons were performed to examine individual main or interaction effects (Winer, 1971).

Behavioral data collected during the Novel Cage test failed tests of homogeneity of variance. Consequently, we used pairwise Mann-Whitney $U$ contrasts to examine NR and MR differences in the behavior.

\section{RESULTS}

\section{Behavior}

Group differences in social and motor behavior were significant and persisted from 18 to 36 months of age. In particular, NR monkeys spent significantly less time engaged in affiliative social (primarily allogrooming and sitting in close contact) behaviors $(\mathrm{F}(1,10)=13.17, p<0.05$; Figure 1, left). The number of affiliative behaviors expressed depended on partner type $(\mathrm{F}(2,20)=4.27, p<0.05)$ with the highest rate found for an HC partner and lowest for a uNR partner (data not shown). NR monkeys showed increased frequency of aggressive (primarily wrestling and pursuit) behaviors $(\mathrm{F}(1,10)=4.60, p=0.05$; Figure 1 , middle) but comparable frequencies of defensive behaviors (Figure 1, right) compared to MR monkeys. Neither aggressive nor defensive behaviors were influenced by partner type. The rate of expression of defensive (primarily escape and defensive crouch) behaviors did increase with age $(\mathrm{F}(2,20)=8.20, p<0.05)$ with the largest increase measured from 18 to 24 months in both NR and MR monkeys.

NR monkeys spent significantly more time engaged in solitary behaviors $(\mathrm{F}(1,10)=4.55, p<0.05$; Figure 2, middle) and NR but not MR monkeys also reliably expressed persistent, abnormal repetitive behaviors $(\mathrm{F}(1,10)=7.418, p<0.05 ;$ Figure 2, left $)$. The latter were present throughout the study.

Measures of walking and running (Figure 2, right) did not differ between rearing groups although time spent engaged in motor activity did decrease with age $(\mathrm{F}(2,20)=32.04$, $p<0.05)$. 
Affiliative Behaviors

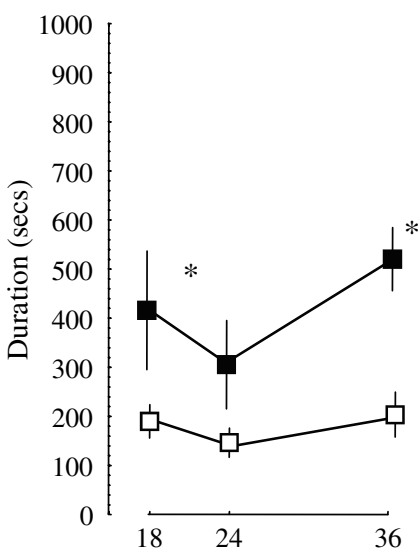

Aggressive Behavior

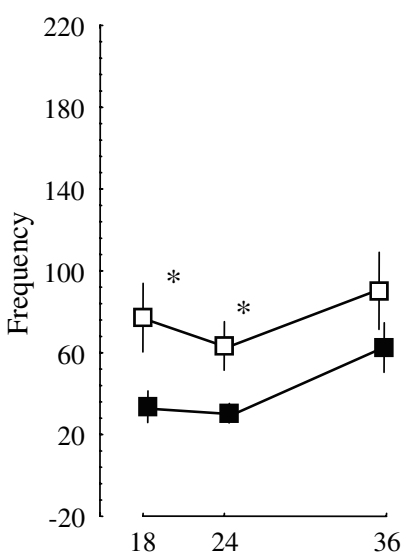

Defensive Behaviors

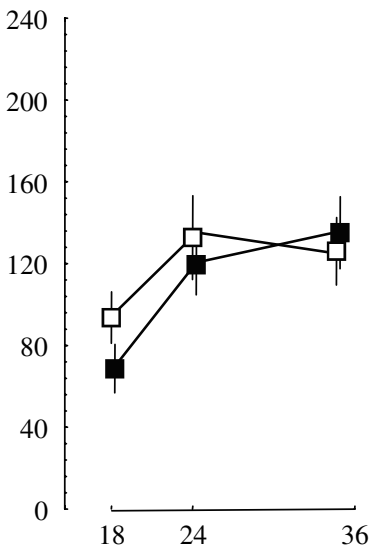

Figure I Social behaviors in MR and NR monkeys. Data represent the measures (mean \pm I SEM) of social activity including the amount of time engaged in affiliative behavior (left), and frequencies of aggressive (middle) and defensive postures (right) during three I-h play sessions scheduled at I8, 24, and 36 months of age. Open symbols represent the social initiatives of NR monkeys. Closed symbols represent the social initiatives of MR monkeys. Asterisks indicate differences $(p<0.05)$ determined by Neuman-Keuls contrasts following repeated measures of ANOVA.
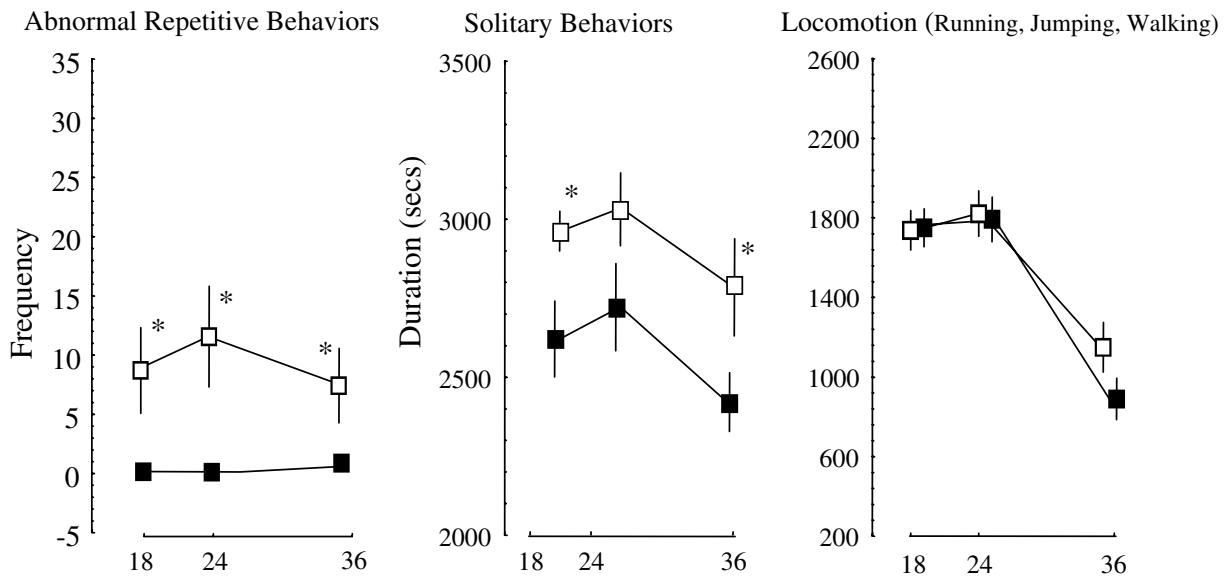

Figure 2 Nonsocial behaviors in MR and NR monkeys. Data represent measures of nonsocial motor behaviors (mean \pm I SEM) including the frequency of stereotyped or abnormal repetitive behavior (left); time spent engaged in solitary behaviors (middle); and walking, running, or jumping between perches (right) during three I-h play sessions scheduled at 18, 24, and 36 months of age. Open symbols represent NR monkeys; closed symbols MR monkeys. Asterisks indicate differences $(p<0.05)$ determined by Neuman-Keuls contrasts following repeated measures of ANOVA.

\section{Basal OT, AVP, and Cortisol}

NR monkeys had significantly decreased levels of CSF OT compared to MR monkeys $(\mathrm{F}(1,20)=14.53, p<0.05)$ at 18 , 24 , and 36 months. No significant differences or interactions were detected for age or rearing history (Figure 3, left). CSF AVP levels measured at 18 and 24 months did not differ according to rearing history or age (levels were not determined at 36 months), nor were there significant interactions detected between age and rearing history (Figure 3, right). Plasma OT levels measured at 36 months of age did not significantly vary by rearing history (Figure 3 , middle), nor did plasma OT values correlate with CSF levels collected in the same session (data not shown).

We also examined the correlation of individual CSF OT levels with the expression of affiliative behavior during the round-robin test at each age. OT levels were correlated with time spent engaged in affiliative social behavior at each of the observation sessions (18 months: $r=0.517, p=0.08 ; 24$ months: $r=0.641, p<0.05$; 36 months: $r=0.814, p<0.05$ ). AVP significantly and negatively correlated with elements of fearful behaviors including frequency of fear grimaces at 18 months $(r=-0.718, p<0.05)$ but not 24 months of age.

Basal levels of plasma cortisol significantly decreased according to age $(\mathrm{F}(2,20)=7.54, p<0.05)$ but not rearing history nor was significant interaction detected between age and rearing history. Basal plasma cortisol levels did not correlate with elements of social or nonsocial behavior, nor were significant correlations detected between CSF levels of OT, AVP, and plasma levels of cortisol.

\section{Novel Cage Stress Response and Social Buffering}

During the novel cage test without a companion, no significant differences were detected between the behaviors expressed by NR and MR monkeys. MR and NR monkeys displayed comparable levels of abnormal repetitive behaviors in the alone condition. Both groups showed compar- 

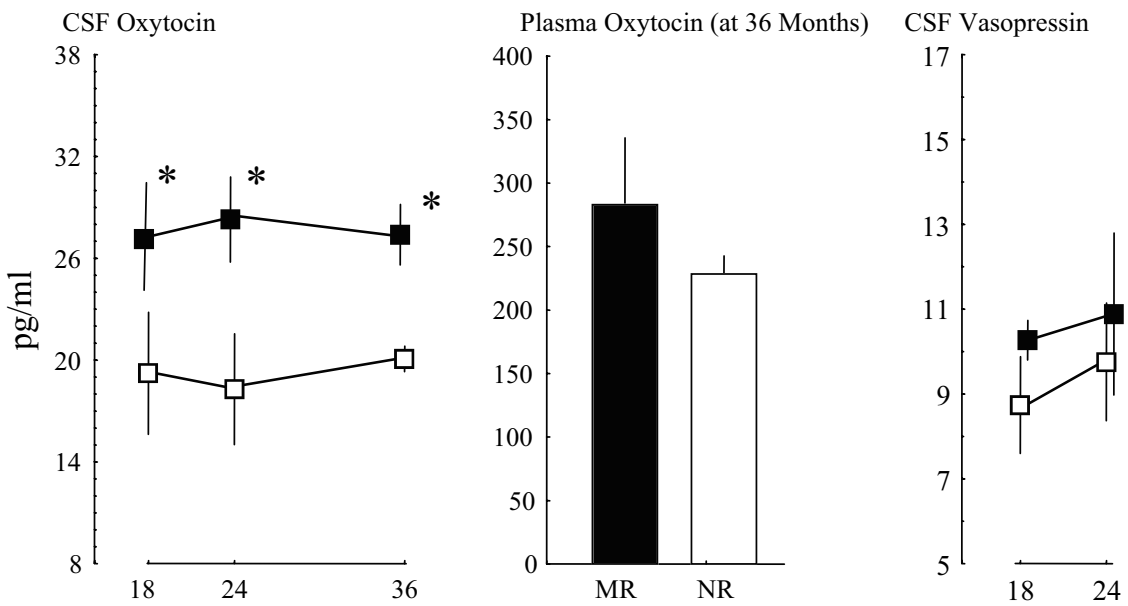

Figure 3 OT and AVP in MR and NR monkeys. Data represent measures of OT and AVP (mean pg/ml \pm I SEM) in NR (open symbols and bars) and MR monkeys (closed symbols and bars) at several ages during the study. The left panel depicts OT detected in CSF at I8, 24 , and 36 months of age. At 36 months we examined OT levels in plasma collected during the same session as CSF samples (middle panel). Also depicted are CSF AVP levels (right panel) measured at 18 and 24 months. Asterisks indicate differences $(p<0.05)$ determined by Neuman-Keuls contrasts following three-way ANOVA with repeated measures for age.

Abnormal/Repetitive Social Contact Behaviors

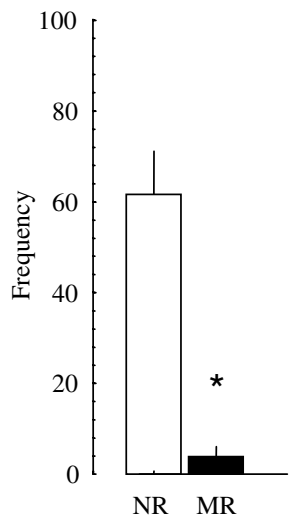

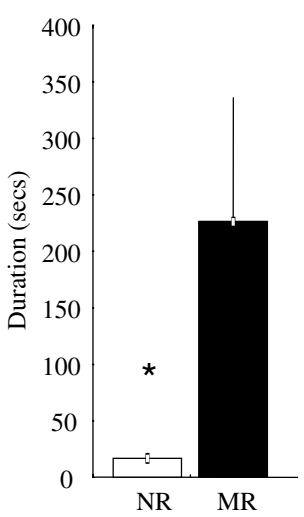

Cortisol Elevation in a Provocative Setting with or without a companion

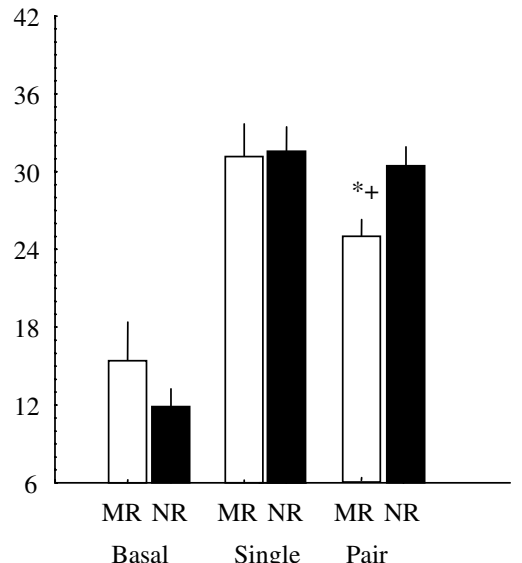

Figure 4 Response to a stressor at 36 months. Data represent behavioral and endocrine responses of NR (open symbols and bars) and MR monkeys (closed symbols and bars) to a 30-min novel cage test with (pair) and without (single) a familiar social companion. The left panel represents the mean ( \pm I SEM) frequencies of abnormal/repetitive behavior. The middle panel depicts the mean ( \pm I SEM) time engaged in social contact behavior. Plasma levels depicted in the right panel represent samples collected within $5 \mathrm{~min}$ of capture (basal), or after $30 \mathrm{~min}$ in a novel cage with and without a social companion. Asterisks represent $p<0.05$ determined by Mann-Whitney $U$ comparisons. For cortisol, asterisks represent cortisol differences $(p<0.05)$ between single and pair conditions measured by paired t-tests. Plus symbols represent differences $(p<0.05)$ between NR and MR monkeys within condition measured by independent $t$-test.

able, elevated levels of cortisol after a 30 min test, but no significant differences in CSF OT or AVP compared to their basal levels. During the test with a companion, all monkeys vocalized less and spent less time self-grooming compared to the alone condition. In the paired condition, NR monkeys had more repetitive behavior $(U=0, z=2.56, p<0.05$; Figure 4, left) and less social behavior $(U=0, z=2.56$, $p<0.05$; Figure 4 , middle) compared to MR monkeys. MR but not NR monkeys showed a modest but significant reduction in cortisol elevation if a familiar companion was present $(t(5)=2.15, p<0.04$ (one-tailed); Figure 4, right). No differences in CSF OT were associated with the presence of a companion during testing compared to the alone condition, although NR monkeys had significantly lower OT levels compared to MR monkeys independent of the testing condition $(\mathrm{F}(1,10)=4.08, p<0.05)$.

\section{DISCUSSION}

As expected from previous studies, monkeys removed from their mother shortly after birth and raised in standard nursery conditions develop a syndrome characterized by decreased affiliation, increased aggression, and increased self-directed, repetitive behavior. This study tested three hypotheses with the following results. First, NR monkeys 
had decreased levels of CSF OT through 36 months of age. CSF OT (but not plasma OT or CSF AVP) correlated with affiliative behavior independent of the rearing condition, whereas CSF AVP correlated negatively with fearful behaviors. Second, basal plasma cortisol measures were not different between the rearing conditions and did not reliably correlate with measures of social interactions. Thirdly, MR but not NR monkeys appeared to benefit from the presence of a familiar companion during a stressprovoking challenge. Consistent with differences in affiliative behavior, MR but not NR monkeys showed an increase in social contact, a reduction in abnormal repetitive behaviors, and a reduced cortisol response in the presence of a familiar companion. Taken together, these results suggest that rearing conditions have long-term effects on both social behavior and central OT concentrations. Several investigators have described the emergence of social deficits in rhesus monkeys following maternal loss and subsequent rearing in standard primate nursery care (Chamove et al, 1973; Erwin et al, 1973; Sackett, 1967, 1972). The deficits appear less severe than those associated with rearing in social isolation, but nevertheless reflect significant and persistent impairment in social, cognitive, and emotional behavior for these animals (Champoux et al, 1992; Sackett, 1967; Suomi, 1991). Social deficits appear to be only partially reversible with intensive socialization with normally reared peers and appear to present a significant risk factor for impaired performance in stress-provocative environments (Champoux et al, 1991; Fahlke et al, 2000; Shannon et al, 1998). Our findings are consistent with previous reports and confirm that deficits in positive social interactions by NR monkeys are particularly evident in reciprocal behaviors such as allogrooming and intermale mounting.

The mechanisms by which early social deprivation results in subsequent social deficits are largely unknown. Just as early visual experience appears critical for the development of normal visual acuity, one might hypothesize that social experience early in development modulates the development of neural pathways required for subsequent social behavior. Indeed, several recent studies in rats have described the long-term neural as well as behavioral consequences of variations in early social experience. Reduced maternal grooming and licking is associated with significant and persistent differences in the adult offspring's emotionality (Caldji et al, 1998), cognition (Zaharia et al, 1996), hypothalamic CRH, and AVP mRNA expression (Liu et al, 1997), as well as binding to $\mathrm{GABA}_{\mathrm{A}}, \mathrm{CRH}, \alpha_{2}$ adrenergic (Caldji et al, 1998), and glucocorticoid receptors in the brain (Sutanto et al, 1996). Two independent groups have reported a decrease in OT receptor binding in the amygdala, following repeated mother-infant separations or decreased maternal licking and grooming (Francis et al, 2000; Noonan et al, 1994).

While these studies in rats have demonstrated the importance of licking and grooming, the proximate factors contributing to abnormal social development in primates are less clear. A variety of extrinsic and intrinsic factors have been suggested, including absence of tactile comfort (Harlow, 1958), lack of vestibular stimulation (Sackett, 1965), loss of control (Mineka et al, 1986), maternal regulation of development (Hofer, 1996), and impaired social cognition (Anderson and Mason, 1978; Capitanio and Mason, 2000). Of course, our NR and MR groups differed in many aspects of rearing. In addition to remaining with their mothers, the MR group was raised outdoors in a complex seminaturalistic environment with considerably more sensory stimulation in their first year relative to monkeys in the NR condition. Various nonsocial aspects of the MR $v s$ NR environments may have contributed to the observed differences: outdoor housing, light cycles, and environmental complexity. However, recent results with brief maternal separations of monkeys housed under seminaturalistic conditions have found similar deficits in affiliative behaviors (and CSF OT), suggesting that the main factor contributing to the observed MR-NR differences was not a general difference in the rearing environment, but a specific loss of maternal care (authors' unpublished data). Nevertheless, the differential contributions of social or other factors (eg reliability of social support or chronic/repeated stress) to the phenotype obtained here remain to be determined and are the subject of ongoing studies.

Is the reduction in CSF OT related to the social deficits in the NR group? Several lines of evidence have linked central OT pathways to social cognition and social motivation. OT appears to facilitate the initiation of maternal care in rats and sheep (reviewed in Insel and Young, 2000), the development of pair bonds in prairie voles (Insel and Hulihan, 1995), and the development of social memory in mice (Ferguson et al, 2000). Mice with a deficiency of OT have a profound and selective social amnesia, which can be reversed by central OT injections (Ferguson et al, 2001). Although OT has been shown to be important in learning about maternal-associated cues in rat pups (Nelson and Panksepp, 1996), there is little previous evidence that social interaction during development alters OT synthesis or OT release.

In addition to our data suggesting that central OT pathways in the rhesus monkey may be affected by differential rearing histories, a similar association between OT CSF levels and social behavioral traits has recently been described in a comparative study of pigtail and bonnet macaques (Rosenblum et al, 2002). The authors report that the more gregarious bonnet macaques have consistently higher levels of CSF OT and lower levels of CRH compared to less affiliative pigtail macaques.

In contrast to some (Higley et al, 1992) but not all (Clarke, 1993; Meyer and Bowman, 1972; Shannon et al, 1998) previous studies, our NR monkeys did not have elevated cortisol levels. The absence of a group difference in basal or poststress cortisol may reflect our limited sampling procedure or age-related differences. We used single point samples under three conditions and did not assess the dynamics of the cortisol response to stress, nor did we assess the response to an endocrine (eg metyrapone) challenge. In addition, to accommodate relatively rapid collection of CSF samples corresponding to plasma samples, animals are anesthetized for biofluid collection. While blood samples were collected within $5 \mathrm{~min}$ of contact and injection, a potential contribution of anesthetic to our endocrine measures cannot be ruled out. A significant, age-dependent decrease in plasma cortisol did emerge in both NR and MR monkeys, and compares with results recently described by Champoux and Suomi (unpublished observations). 
One of the benefits of strong social relations is the protection they appear to provide against the adverse consequences of stress (Cobb, 1976). Efforts to examine social buffering of stress in animals, much of it in nonhuman primates, has recently been summarized in a review by Levine (2000). Initial evidence of social buffering emerged in studies of highly provocative mother-infant separations in squirrel monkeys. When an infant squirrel monkey is removed from its mother it shows a profound stress response. This response can be significantly reduced if the infant remains in a familiar environment with familiar companions (Wiener et al, 1987). According to Levine, the critical variable for this effect is the presence of familiar companions. The stress-reducing properties of familiar companions persist into adulthood in squirrel monkeys, but appears to require the presence of more than a single companion (Coe et al, 1982; Vogt et al, 1981); however see also Hennessy (1984). Studies in rhesus (Gunnar et al, 1980; Gust et al, 1994) and bonnet (Boccia et al, 1997) macaques describe similar stress-reducing benefits of social companions and suggest that for some measures of stress (T-cell suppression) a single familiar companion can effectively buffer stress effects. These data provide strong evidence that social companions can effectively modulate the effects of stress in nonhuman primates. Remarkably, little is known about the interaction of postnatal rearing experience and the capacity of familiar companions to modulate stress. Hennessy (1984) describes evidence of social buffering in squirrel monkeys reared on inanimate maternal surrogates. Notably this effect was achieved under conditions where social buffering was ineffective in MR monkeys.

In the current studies, NR monkeys appear to have a relative deficit in the capacity to use social support in a provocative situation. This was expressed both in the decreased amount of social contact with a companion, elevated stereotypies, and elevated cortisol response compared to MR monkeys. Whether this deficit represents a consequence of reduced OT remains to be determined. Several studies have described the potential anxiolytic or antistress effects of central OT administration in rats (Uvnas-Moberg et al, 1994), mice (McCarthy, 1996; McCarthy et al, 1996), and squirrel monkeys (Winslow and Insel, 1991a,b). However, it is not clear that these exogenous elevations of OT are related to differences in endogenous OT described here. There are several reports relating CSF and plasma levels of OT to human psychopathologies including OCD (Leckman et al, 1994), anorexia and depression (Frank et al, 2000), and Prader Willi syndrome (Martin et al, 1998). Differing basal levels and modulation of plasma OT levels have also been associated with differing levels of emotionality (Turner et al, 1999). The data presented here suggest that OT process may also contribute to the stress-protective effects of social support in humans (Coyne and Downey, 1991; Greenwood et al, 1996; Vilhjalmsson, 1993).

The behavioral profile of the NR monkeys (lack of reciprocal behavior, stereotypies, decreased affiliation) resembles some of the characteristic features of autism. Although there are no data on CSF OT in autism, plasma OT is reduced markedly in children with autism (Modahl et al, 1998), apparently because of a problem with C-terminal processing of the immature peptide (Green et al, 2001).
While these results suggest that autism may involve a mutation or variation in one of the post-translational processing enzymes for OT, our results show a relation between decreased CSF OT and social deficits based on an environmental not a genetic intervention. Ongoing studies examine the differential contribution of social and other environmental factors to social and emotional development, and extend these manipulations to both male and female monkeys.

In summary, CSF OT levels were persistently reduced in male rhesus monkeys with significant social deficits. OT levels were positively related to the expression of affiliative social behaviors. In a novel environment, MR but not NR monkeys appeared to derive buffering from a social companion. Taken together these data suggest that abnormal rearing influences the normal development of brain OT systems and social competence in rhesus monkeys.

\section{ACKNOWLEDGMENTS}

This work was supported in part by the National Institute of Mental Health Grants MH57704 and MH58922 to JTW, and National Science Foundation Contract No. IBN9876754 to Emory University, and by Grant RR-00165 from the NIH/ NCRR to the Yerkes Regional Primate Research Center. The Yerkes Primate Center is fully accredited by the American Association for Accreditation of Laboratory Animal Care.

\section{REFERENCES}

Altmann J (1974). Observational study of behavior: sampling methods. Behaviour 49: 227-267.

Altmann SA (1962). A field study of the sociobiology of rhesus monkeys. Ann NY Acad Sci 102: 338-435.

Anderson CO, Mason WA (1978). Competitive social strategies in groups of deprived and experienced rhesus monkeys. Dev Psychobiol 11: 289-299.

Boccia ML, Scanlan JM, Laudenslager ML, Berger CL, Hijazi AS, Reite ML (1997). Juvenile friends, behavior, and immune responses to separation in bonnet macaque infants. Physiol Behav 61: 191-198.

Caldji C, Tannenbaum B, Sharma S, Francis D, Plotsky PM, Meaney MJ (1998). Maternal care during infancy regulates the development of neural systems mediating the expression of fearfulness in the rat. Proc Natl Acad Sci USA 95: 5335-5340.

Capitanio JP, Mason WA (2000). Cognitive style: problem solving by rhesus macaques (Macaca mulatta) reared with living or inanimate substitute mothers. J Comp Psychol 114: 115-125.

Chamove AS, Rosenblum LA, Harlow HF (1973). Monkeys (Macaca mulatta) raised only with peers. A pilot study. Anim Behav 21: 316-325.

Champoux M, Byrne E, DeLizio R, Suomi SJ (1992). Motherless mothers revisited: rhesus maternal behavior and rearing history. Primates 33: 251-255.

Champoux M, Metz B, Suomi SJ (1991). Behavior of nursery/peerreared and mother-reared rhesus monkeys from birth through 2 years of age. Primates 32: 509-514.

Clarke AS (1993). Social rearing effects on HPA axis activity over early development and in response to stress in rhesus monkeys. Dev Psychobiol 26: 433-446.

Cobb S (1976). Presidential Address-1976. Social support as a moderator of life stress. Psychosom Med 38: 300-314.

Coe CL, Franklin D, Smith ER, Levine S (1982). Hormonal responses accompanying fear and agitation in the squirrel monkey. Physiol Behav 29: 1051-1057. 
Coyne JC, Downey G (1991). Social factors and psychopathology: stress, social support, and coping processes. Annu Rev Psychol 42: 401-425.

Erwin J, Mitchell G, Maple T (1973). Abnormal behavior in nonisolate-reared rhesus monkeys. Psychol Rep 33: 515-523.

Fahlke C, Lorenz JG, Long J, Champoux M, Suomi SJ, Higley JD (2000). Rearing experiences and stress-induced plasma cortisol as early risk factors for excessive alcohol consumption in nonhuman primates. Alcohol Clin Exp Res 24: 644-650.

Ferguson JN, Aldag JM, Insel TR, Young LJ (2001). Oxytocin in the medial amygdala is essential for social recognition in the mouse. J Neurosci 21: 8278-8285.

Ferguson JN, Young LJ, Hearn EF, Matzuk MM, Insel TR, Winslow JT (2000). Social amnesia in mice lacking the oxytocin gene. Nat Genet 25: 284-288.

Francis DD, Champagne FC, Meaney MJ (2000). Variations in maternal behaviour are associated with differences in oxytocin receptor levels in the rat. J Neuroendocrinol 12: 1145-1148.

Frank GK, Kaye WH, Altemus M, Greeno CG (2000). CSF oxytocin and vasopressin levels after recovery from bulimia nervosa and anorexia nervosa, bulimic subtype. Biol Psychiatry 48: 315-318.

Ginsberg SD, Hof PR, McKinney WT, Morrison JH (1993a). The noradrenergic innervation density of the monkey paraventricular nucleus is not altered by early social deprivation. Neurosci Lett 158: 130-134.

Ginsberg SD, Hof PR, McKinney WT, Morrison JH (1993b). Quantitative analysis of tuberoinfundibular tyrosine hydroxylase- and corticotropin-releasing factorimmunoreactive neurons in monkeys raised with differential rearing conditions. Exp Neurol 120: 95-105.

Green L, Fein D, Modahl C, Feinstein C, Waterhouse L, Morris M (2001). Oxytocin and autistic disorder: alterations in peptide forms. Biol Psychiatry 50: 609-613.

Greenwood DC, Muir KR, Packham CJ, Madeley RJ (1996). Coronary heart disease: a review of the role of psychosocial stress and social support. J Public Health Med 18: 221-231.

Gunnar MR, Gonzalez CA, Levine S (1980). The role of peers in modifying behavioral distress and pituitary-adrenal response to a novel environment in year-old rhesus monkeys. Physiol Behav 25: 795-798.

Gust DA, Gordon TP, Brodie AR, McClure HM (1994). Effect of a preferred companion in modulating stress in adult female rhesus monkeys. Physiol Behav 55: 681-684.

Hall FS, Huang S, Fong GW, Pert A, Linnoila M (1998). Effects of isolation-rearing on locomotion, anxiety and responses to ethanol in Fawn Hooded and Wistar rats. Psychopharmacology (Berl) 139: 203-209.

Harlow HF (1958). The nature of love. Am Psychologist 1: 673-685.

Harlow HF, Dodsworth RO, Harlow JK (1955). Total social isolation in monkeys. Proc Natl Acad Sci 54: 90-97.

Harlow HF, Harlow MK (1962). The effect of rearing conditions on behavior. Bull Menninger Clin 26: 213-224.

Harlow HF, Suomi SJ (1974). Induced depression in monkeys. Behav Biol 12: 273-296.

Hennessy MB (1984). Presence of companion moderates arousal of monkeys with restricted social experience. Physiol Behav 33: 693-698.

Higley JD, Suomi SJ, Linnoila M (1992). A longitudinal assessment of CSF monoamine metabolite and plasma cortisol concentrations in young rhesus monkeys. Biol Psychiatry 32: 127-145.

Hinde RA, Spencer-Booth Y (1967). The behaviour of socially living rhesus monkeys in their first two and a half years. Anim Behav 15: 169-196.

Hofer MA (1996). On the nature and consequences of early loss. Psychosom Med 58: 570-581.

Immelmann K (1969). On the effect of early experience upon sexual object fixation in estrildine finches. $Z$ Tierpsychol 26: $677-691$
Insel TR (1992). Oxytocin-a neuropeptide for affiliation: evidence from behavioral, receptor autoradiographic, and comparative studies. Psychoneuroendocrinology 17: 3-35.

Insel TR, Hulihan TJ (1995). A gender-specific mechanism for pair bonding: oxytocin and partner preference formation in monogamous voles. Behav Neurosci 109: 782-789.

Insel TR, Young LJ (2000). Neuropeptides and the evolution of social behavior. Curr Opin Neurobiol 10: 784-789.

Leckman JF, Goodman WK, North WG, Chappell PB, Price LH, Pauls DL et al (1994). Elevated cerebrospinal fluid levels of oxytocin in obsessive-compulsive disorder. Comparison with Tourette's syndrome and healthy controls. Arch Gen Psychiatry 51: 782-792.

Levine S (2000). Influence of psychological variables on the activity of the hypothalamic-pituitary-adrenal axis. Eur J Pharmacol 405: $149-160$

Liu D, Caldji C, Sharma S, Plotsky PM, Meaney MJ (2000). Influence of neonatal rearing conditions on stress-induced adrenocorticotropin responses and norepinepherine release in the hypothalamic paraventricular nucleus. J Neuroendocrinol 12: 5-12.

Liu D, Diorio J, Tannenbaum B, Caldji C, Francis D, Freedman A et al (1997). Maternal care, hippocampal glucocorticoid receptors, and hypothalamic-pituitary-adrenal responses to stress. Science 277: 1659-1662.

Martin A, State M, Anderson GM, Kaye WM, Hanchett JM, McConaha CW et al (1998). Cerebrospinal fluid levels of oxytocin in Prader-Willi syndrome: a preliminary report. Biol Psychiatry 44: 1349-1352.

McCarthy MM (1996). Estrogen modulation of oxytocin and its relation to behavior. Adv Exp Med Biol 395: 235-246.

McCarthy MM, McDonald CH, Brooks PJ, Goldman AD (1996). An anxiolytic action of oxytocin is enhanced by estrogen in the mouse. Physiol Behav 60: 1209-1215.

Meyer JS, Bowman RE (1972). Rearing experience, stress and adrenocorticosterioids in the rhesus monkey. Physiol Behav 8: 339-343.

Mineka S, Gunnar M, Champoux M (1986). Control and early socioemotional development: infant rhesus monkeys reared in controllable versus uncontrollable environments. Child Dev 57: 1241-1256.

Modahl C, Green L, Fein D, Morris M, Waterhouse L, Feinstein C, et al (1998). Plasma oxytocin levels in autistic children. Biol Psychiatry 43: 270-277.

Nelson E, Panksepp J (1996). Oxytocin mediates acquisition of maternally associated odor preferences in preweanling rat pups. Behav Neurosci 110: 583-592.

Noonan LR, Caldwell JD, Li L, Walker CH, Pedersen CA, Mason GA (1994). Neonatal stress transiently alters the development of hippocampal oxytocin receptors. Brain Res Dev Brain Res 80: 115-120.

Reinhardt V, Reinhardt A, Bercovitch FB, Goy RW (1986). Does intermale mounting function as a dominance demonstration in rhesus monkeys? Folia Primatol (Basel) 47: 55-60.

Rosenblum LA, Smith EL, Altemus M, Scharf BA, Owens MJ, Nemeroff CB et al (2002). Differing concentrations of corticotropin-releasing factor and oxytocin in the cerebrospinal fluid of bonnet and pigtail macaques. Psychoneuroendocrinology 27: 651-660.

Rutter M, Andersen-Wood L, Beckett C, Bredenkamp D, Castle J, Groothues C et al (1999). Quasi-autistic patterns following severe early global privation. English and Romanian Adoptees (ERA) Study Team. J Child Psychol Psychiatry 40: 537-549.

Sackett GP (1965). Effects of rearing conditions upon the behavior of rhesus monkeys (Macaca mulatta). Child Dev 36: 855-868.

Sackett GP (1967). Some persistent effects of different rearing conditions on preadult social behavior of monkeys. J Comp Physiol Psychol 64: 363-365. 
Sackett GP (1972). Exploratory behavior of rhesus monkeys as a function of rearing experiences and sex. Dev Psychol 6: $260-270$.

Sackett GP (1984). A nonhuman primate model of risk for deviant development. Am J Mental Deficiency 88: 469-476.

Sanchez MM, Hearn EF, Do D, Rilling JK, Herndon JG (1998). Differential rearing affects corpus callosum size and cognitive function of rhesus monkeys. Brain Res 812: 38-49.

Shannon C, Champoux M, Suomi SJ (1998). Rearing condition and plasma cortisol in rhesus monkey infants. Am J Primatol 46: 311-321.

Siegel SJ, Ginsberg SD, Hof PR, Foote SL et al (1993). Effects of social deprivation in prepubescent rhesus monkeys: immunohistochemical analysis of the neurofilament protein triplet in the hippocampal formation. Brain Res 619: 299-305.

Suomi SJ (1991). Early stress and adult emotional reactivity in rhesus monkeys. Ciba Found Symp 156: 171-183; discussion 183-188.

Suomi SJ, Harlow HF, Kimball SD (1971). Behavioral effects of prolonged partial social isolation in the rhesus monkey. Psychol Rep 29: 1171-1177.

Sutanto W, Rosenfeld P, de Kloet ER, Levine S (1996). Long-term effects of neonatal maternal deprivation and ACTH on hippocampal mineralocorticoid and glucocorticoid receptors. Brain Res Dev Brain Res 92: 156-163.

Turner RA, Altemus M, Enos T, Cooper B, McGuinness T (1999). Preliminary research on plasma oxytocin in normal cycling women: investigating emotion and interpersonal distress. Psychiatry 62: 97-113.

Uvnas-Moberg K, Ahlenius S, Hillegaart V, Alster P (1994). High doses of oxytocin cause sedation and low doses cause an anxiolytic-like effect in male rats. Pharmacol Biochem Behav 49: $101-106$.

Vilhjalmsson R (1993). Life stress, social support and clinical depression: a reanalysis of the literature. Soc Sci Med 37: 331-342.

Vogt JL, Coe CL, Levine S (1981). Behavioral and adrenocorticoid responsiveness of squirrel monkeys to a live snake: is flight necessarily stressful? Behav Neural Biol 32: 391-405.

Wiener SG, Johnson DF, Levine S (1987). Influence of postnatal rearing conditions on the response of squirrel monkey infants to brief perturbations in mother-infant relationships. Physiol Behav 39: 21-26.

Winer BJ (1971). Statistical principles in experimental design. New York: McGraw-Hill.

Winslow J, Insel TR (1991a). Vasopressin modulates male squirrel monkeys' behavior during social separation. Eur J Pharmacol 200: 95-101.

Winslow JT, Insel TR (1991b). Social status in pairs of male squirrel monkeys determines the behavioral response to central oxytocin administration. J Neurosci 11: 2032-2038.

Zaharia MD, Kulczycki J, Shanks N, Meaney MJ, Anisman H (1996). The effects of early postnatal stimulation on Morris water-maze acquisition in adult mice: genetic and maternal factors. Psychopharmacology (Berl) 128: 227-239. 